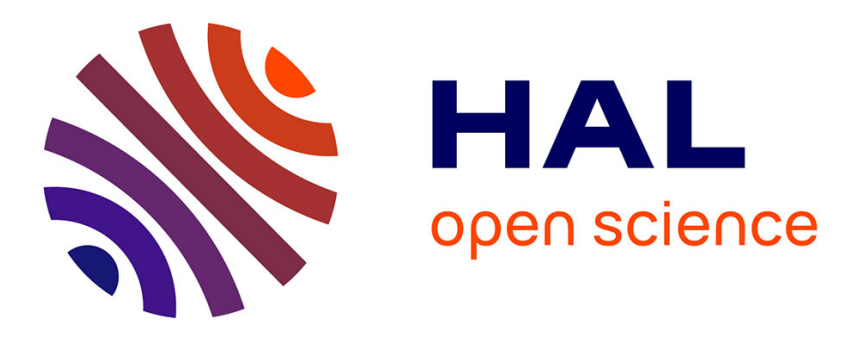

\title{
Structural differences in eating disorder psychopathology after history of childhood abuse: Insights from a Bayesian network analysis.
}

Rachel Rodgers, Russell Dubois, Sylvain Thiébaut, Isabelle Jaussent, Laurent Maïmoun, Maude Sénèque, Patrick Lefebvre, Eric Renard, Philippe Courtet, Sébastien Guillaume

\section{To cite this version:}

Rachel Rodgers, Russell Dubois, Sylvain Thiébaut, Isabelle Jaussent, Laurent Maïmoun, et al.. Structural differences in eating disorder psychopathology after history of childhood abuse: Insights from a Bayesian network analysis.. Journal of Abnormal Psychology, 2019, 128 (8), pp.795-805. 10.1037/abn0000470 . hal-02313569

\section{HAL Id: hal-02313569 \\ https://hal.science/hal-02313569}

Submitted on 19 Jun 2020

HAL is a multi-disciplinary open access archive for the deposit and dissemination of scientific research documents, whether they are published or not. The documents may come from teaching and research institutions in France or abroad, or from public or private research centers.
L'archive ouverte pluridisciplinaire HAL, est destinée au dépôt et à la diffusion de documents scientifiques de niveau recherche, publiés ou non, émanant des établissements d'enseignement et de recherche français ou étrangers, des laboratoires publics ou privés. 


\title{
Structural Differences in Eating Disorder Psychopathology After History of Childhood Abuse: Insights From a Bayesian Network Analysis
}

\author{
Rachel F. Rodgers \\ Northeastern University and Lapeyronie Hospital, Regional \\ University Hospital, Montpellier, France
}

Sylvain Thiebaut

Lapeyronie Hospital, Regional University Hospital, Montpellier, France

\section{Laurent Maimoun}

Montpellier University Hospital, Montpellier, France, and Montpellier University

Patrick Lefebvre

Montpellier University Hospital, Montpellier, France

\author{
Russell DuBois \\ Northeastern University
}

Isabelle Jaussent

Inserm U1061, Montpellier, France
Maude Seneque

Lapeyronie Hospital, Regional University Hospital, Montpellier, France, and Inserm U1061, Montpellier, France

Eric Renard

Montpellier University Hospital, Montpellier, France, and University of Montpellier

Philippe Courtet and Sébastien Guillaume

Lapeyronie Hospital, Regional University Hospital, Montpellier, France, and Inserm U1061, Montpellier, France

\begin{abstract}
Childhood abuse is frequent among individuals with eating disorders and is associated with complex clinical presentations. However, to date, the differences in the presentations of eating disorders between these groups are poorly understood. The present study employed a Bayesian network approach to model the interactive network structure of eating disorder psychopathology, and to investigate the differences in symptom importance and network structure between individuals with eating disorders with and without an experience of childhood abuse in a sample 327 treatment-seeking individuals. Among individuals with a history of childhood abuse, a specific 4-symptom pathway emerged, leading from overvaluation of shape and weight and ending in overeating (overvaluation of weight and shape $\rightarrow$ loss of control $\rightarrow$ depressed mood $\rightarrow$ overeating). Loss of control eating and depressed mood emerged as the more important driving symptoms. In contrast, the eating disorder symptom network among the group with no abuse was organized around a heightened investment in weight and shape, and resulting efforts to control or alter weight and shape through dieting and exercise behaviors. The symptoms with the highest importance in this nonabuse group were overeating and overvaluation of weight and shape. These results support the existence of a distinct eating disorder symptom network characteristic of individuals with a history of childhood trauma, and add to the hypotheses of a maltreated eco-phenotype in eating disorders. They may be also inform treatment target in abused people with eating disorders.
\end{abstract}

\footnotetext{
Rachel F. Rodgers, Applied Psychology Program for Eating and Appearance Research, Department of Applied Psychology, Northeastern University, and Department of Psychiatric Emergency and Acute Care, Lapeyronie Hospital, Regional University Hospital, Montpellier, France; Russell DuBois, Applied Psychology Program for Eating and Appearance Research, Department of Applied Psychology, Northeastern University; Sylvain Thiebaut, Department of Psychiatric Emergency and Acute Care, Lapeyronie Hospital, Regional University Hospital; Isabelle Jaussent, Inserm U1061, Montpellier, France; Laurent Maimoun, Department of Nuclear Medicine, Montpellier University Hospital, Montpellier, France, and PhyMedExp, INSERM, CNRS, Montpellier University; Maude Seneque, Department of Psychiatric Emergency and Acute Care, Lapeyronie Hospital, Regional University Hospital, and Inserm U1061; Patrick
}

Lefebvre, Department of Endocrinology, Diabetes, and Nutrition, Montpellier University Hospital; Eric Renard, Department of Endocrinology, Diabetes, and Nutrition, Montpellier University Hospital, and Institute of Functional Genomics, University of Montpellier; Philippe Courtet and Sébastien Guillaume, Department of Psychiatric Emergency and Acute Care, Lapeyronie Hospital, Regional University Hospital, and Inserm U1061.

This study received financial support from Regional University Hospital Montpellier (AOI UF 8854). The Regional University Hospital Montpellier had no role in the design, analysis, interpretation, or publication of this study.

Correspondence concerning this article should be addressed to Rachel F. Rodgers, Department of Applied Psychology, Northeastern University, 404 INV, 360 Huntington Avenue, Boston, MA 02115. E-mail: r.rodgers@ northeastern.edu 


\section{General Scientific Summary}

Childhood abuse is frequent among individuals with eating disorders. This study used network analysis to reveal differences in the way that eating disorder symptoms are related to each other among individuals with a childhood abuse history as compared with those without such abuse. Depressed mood emerged as an important driver of eating disorder psychopathology in the subgroup reporting childhood abuse.

Keywords: childhood abuse, depressed mood, eating disorders, loss of control eating, networks

The comorbidity between trauma exposure and eating disorders has been well-established in the extant literature, including the role of childhood abuse and trauma in later onset eating disorders (Brewerton, 2007; Trottier \& MacDonald, 2017). In addition, history of childhood trauma is frequently associated with complex clinical presentations, including earlier onset of eating disorders, and heightened severity (Caslini et al., 2016; Molendijk, Hoek, Brewerton, \& Elzinga, 2017). To date, the characteristics of eating disorders among individuals who have experienced childhood abuse and display these complex presentations are poorly understood (Trottier \& MacDonald, 2017). With the aim of contributing to bridging this gap, the present study investigated differences in the patterns of eating disorders symptoms between individuals with and without a history of childhood abuse using a network approach.

As documented by two recent meta-analyses, childhood trauma and abuse regardless of the subtype (emotional childhood trauma, physical childhood trauma, sexual childhood trauma) are more frequent among individuals with eating disorders, compared with the general population (Caslini et al., 2016; Molendijk et al., 2017). Specifically, the overall odds of having an eating disorder has been estimated to be 3.21 time higher (95\% CI [2.29, 4.51], $p<.001)$ in individuals reporting a childhood trauma. In addition, recent findings have suggested that childhood trauma is associated with increased severity of a wide range of eating disorders features, such as eating, shape, and weight concerns and impaired daily functioning (Guillaume et al., 2016). A history of childhood trauma and abuse has also been associated with poorer long-term outcomes among eating disorder patients, with those reporting childhood trauma exhibiting less improvement in both eatingrelated psychopathology and psychiatric comorbidity 3 years after the completion of cognitive-behavioral therapy (Castellini et al., 2018). Thus, childhood trauma and abuse has been shown to negatively impact the course and severity of eating disorders in multiple ways.

To date, the ways in which eating disorders may differ among individuals with a history of childhood abuse, in terms of the patterns of symptoms and maintenance factors, is unclear. Differences in presentations of eating disorders among this group may be attributable to the ways in which eating disorder symptoms might cluster into specific types of causally linked networks. Several hypothetical pathways, which would result in differences in the network structure, have received preliminary support (Trottier \& MacDonald, 2017). First, related to biological theories, exposure to severe childhood adverse events may be related to biological vulnerabilities or altered epigenetic expressions, which may in turn be related to psychopathology (Steiger et al., 2011; Steiger, La- bonté, Groleau, Turecki, \& Israel, 2013). Childhood abuse has been associated with altered neuroendocrine functioning in women with eating disorders (Monteleone, Monteleone, et al., 2018). Moreover, childhood trauma history has been associated with alterations in brain structures thought to be implicated in eating disorder psychopathology among individuals with anorexia and bulimia nervosa (Monteleone et al., 2017). Taken together, these studies suggest that exposure to childhood abuse may be associated with long-lasting neuro-endocrine modifications, which may account for it increasing risk for psychopathology including eating disorders, and underlie the specificities in the presentation and course of eating disorder pathology among individuals with a history of childhood abuse. Based on these theories, it would be anticipated that differences in the clinical presentations of eating disorders in the aftermath of childhood abuse might be illustrated by differences in the organization of networks of eating disorder symptoms in these patients, and the ways that symptoms are interrelated.

In addition, theories focusing on the psychological factors that may account for the relationships between childhood abuse and later eating disorders have been developed. In particular, escape theory posits that childhood abuse may result deficits in emotion regulation and/or behavioral control, which may contribute to the development and maintenance of eating disorder psychopathology, likely as a maladaptive means of regulating negative emotion (Trottier \& MacDonald, 2017). In particular, among individuals with an abuse history, binge eating has been conceptualized as a maladaptive coping strategy, aiming to regulate negative affect through avoidance, and distraction from trauma reminders (Harrington, Crowther, Payne Henrickson, \& Mickelson, 2006). Similarly, in Anorexia Nervosa, heightened negative arousal in conjunction with difficulties tolerating negative affect have been hypothesized to lead to maladaptive emotional regulation strategies including avoidance and escape (Haynos \& Fruzzetti, 2011). Consistent with these theories, emotional dysregulation has been found to mediate the relationship between childhood trauma exposure and eating pathology (Michopoulos et al., 2015; Moulton, Newman, Power, Swanson, \& Day, 2015). These pathways suggest that specificities in clinical presentations might be reflected in eating disorder symptom networks by the role played by affectrelated symptoms, and particularly negative affect.

Together, these findings support the existence of differential psychological and biological factors impacting eating disorders among individuals with a childhood traumatic experience. However, little is known about the unique symptom-level, phenotypic expression of eating disorder psychopathology among this group. This distinction is particularly important given that theoretical 
models and clinical protocols for eating disorders typically rest on this level of analysis (e.g., understanding and manipulating the causal relationships between ED-related thoughts, feelings, and behaviors in eating disorder focus cognitive-behavioral therapy).

In recent years, the network approach has emerged as a useful framework within which to investigate psychopathology (Borsboom \& Cramer, 2013; McNally, 2016). In contrast to traditional latent trait approaches, the network model conceptualizes psychopathology as a system of causal interactions, in which the symptoms themselves constitute the disorder, rather than arising from an underlying entity (McNally, 2016). Networks consist of nodes that in this context represent symptoms, as well as edges representing the relationships between symptoms. A major contribution of the network approach to psychopathology is the ability to identify highly important symptoms in a given symptom network. Often referred to as symptom centrality, symptoms that are highly influential in the network are thought to represent particularly important constructs in a given mental health syndrome (McNally, 2016). Such an approach has already proved useful in furthering our understanding of psychopathology, including when applied to eating disorders (DuBois, Rodgers, Franko, Eddy, \& Thomas, 2017; Levinson et al., 2017; Rodgers, DuBois, Frumkin, \& Robinaugh, 2018). Overall, the findings from studies examining networks of eating disorder symptoms have provided support for cognitive-behavioral transdiagnostic models of eating disorders that emphasizes the core role of overvaluation of weight and shape in the development of restrictive behaviors, which, in conjunction with negative affect, result in binge-eating and compensatory behaviors (Fairburn, Cooper, \& Shafran, 2003).

Although psychopathology networks are theoretically causal systems, not all analytic approaches allow for the examination of directionality - a critical component in the network approach to psychopathology. Bayesian approaches have been gaining support as a useful tool for examining directed, hence causal, relationships within cross-sectional networks (McNally, Heeren, \& Robinaugh, 2017). In mathematics, a Bayesian network is a probabilistic graphical model that represents a map of the relationships among a set of variables based on their conditional dependencies. Applied to psychopathology, Bayesian networks can be understood as a group of symptoms connected to one another through a specific pattern of predicted relationships.

The addition of directionality into cross-sectional psychopathology network models is particularly relevant given recent concerns regarding the ambiguity when interpreting findings from undirected networks (Fried et al., 2017). An additional strength of these approaches is that they allow for researchers to integrate a priori, theory, or empirically driven knowledge regarding the relationships among the nodes of a network (Heckerman, Geiger, \& Chickering, 1995). As such, the ability to introduce conceptually driven constraints into an otherwise data-driven methodology is of critical importance to the field. In the context of psychopathology, for example, a researcher may limit the array of potential symptom associations to only those that are clinically plausible or established through prior research (McNally, 2016). Bayesian network analysis produces sparse graphs, which are more readily interpretable when compared with the highly connected networks of certain correlation-based analyses. This contribution is particularly relevant when investigating group-level differences in the overall patterns of relationships among symptoms.
Although Bayesian models have been used in a variety of scientific disciplines to estimate the interactive structure of a set of variables from cross-sectional data, they have only recently been successfully utilized in psychopathology research to elucidate the interactive structure of mental health syndromes (Jones, Mair, Riemann, Mugno, \& McNally, 2018; McNally, Mair, Mugno, \& Riemann, 2017; McNally, 2016; McNally et al., 2017). However, their usefulness for elucidating directed relationships within networks makes them particularly suited to for preliminary investigations into potential causal systems of eating disorder symptoms, and thereby investigating some of the ways in which childhood trauma might be associated with differences in the psychopathology of eating disorders. In addition, to date, all of the studies examining networks of eating disorder symptoms have utilized nondirected network analytic approaches, which limits their capacity to contribute to our understanding of the directed relationships within these networks. Bayesian approaches partially overcome some of these limitations, and therefore are of particular interest when using cross-sectional data. Thus, in the present study we utilized a Bayesian network approach to model the interactive network structure of eating disorder psychopathology and to investigate the differences in symptom importance and overall network structure between individuals with eating disorders with and without a past experience of childhood abuse.

Building on the work described above, the present study aimed to investigate the mechanisms by which childhood trauma influences eating disorder. We thus explore the characteristics of eating disorder symptom networks among individuals with and without childhood trauma using a Bayesian network approach. Network differences between these two groups might help explain specificities in clinical presentation and course.

\section{Method}

\section{Participants and Recruitment}

A total of 353 individuals with eating disorders were recruited for the study. After removing individuals who exceeded our threshold for missing data (more than $30 \%$ missing data on relevant measures described below), a final sample of 327 individuals was included in subsequent analyses. As described elsewhere (Guillaume et al., 2016), participants were consecutively recruited from an outpatient eating disorder unit at a university hospital in Montpellier, France. This ancillary study to a larger project was approved by the local ethics committee (CPP Sud Méditerranée IV) and signed, informed consent was obtained from all participants (with parental consent obtained for participants under the age of 18 years). This study received financial support from CHRU Montpellier (AOI UF 8854). The CHRU Montpellier had no role in the design, analysis, interpretation, or publication of this study. The research was conducted according to the tenets of the Declaration of Helsinki. Inclusion criteria were age of 15 years or older, French speaking, and diagnosed with an eating disorders according to Diagnostic and Statistical Manual of Mental Disorders, fifth edition (DSM-5) criteria. Exclusion criteria were absence of an ED, presenting with a physical condition that prevented from participating in the research $(n=8)$, and refusal to participate in the study $(n=17)$. 
The diagnosis of ED was based on the DSM-5 criteria. Patients were assessed by both a senior psychiatrist (S.T. or S.G.) and a senior nutritionist (P.L. or E.R.) experienced in assessment and management of eating disorders, using a nonstructured clinical assessment as well as a structured evaluation with the MiniInternational Neuropsychiatric Interview (MINI, Version 5.0.0, Lecrubier et al., 1997). Patients also completed the Eating Disorders Examination questionnaire (Fairburn \& Beglin, 2008). In addition, information from medical records and from relatives were collected when available. Diagnosis was then established by consensus of the investigators using a best-estimated procedure using information from all these assessments. The sample included $n=167(51 \%)$ patients with a diagnosis of anorexia nervosa, $n=$ $90(27.5 \%)$ with a diagnosis of bulimia nervosa, $n=36(11 \%)$ with a diagnosis of binge eating disorder, and $n=34$ (10.5\%) with unspecified feeding or eating disorders.

\section{Measures}

Eating disorder symptoms. Our process of symptom measurement was largely influenced by the proposed assumptions regarding node selection in Bayesian networks, the impact of node selection on causal inferences (McNally, 2016; Pearl, 2011), and previous network models of eating disorders (e.g., Rodgers et al., 2018). Specifically, we measured eating disorder symptoms with select items from the Eating Disorder Examination Questionnaire (EDE-Q; Fairburn \& Beglin, 2008) When used in its entirety, the full EDE-Q includes 28 items that are scored on a 7-point scale from 0 (no days/not at all) to 6 every day/markedly) with higher scores indicating greater symptomatology.

Depressed mood. We used a single item of the Beck Depression Inventory (BDI; Beck, Steer, \& Carbin, 1988) to assess depressed mood. The full BDI includes 21 items that are scored on a 4-point scale from 0 to 3 , with higher scores indicating greater symptomatology.

When appropriate, we used single items from the EDE-Q and BDI to measure eating disorder symptoms. Single item measurement was used only when the item represented a distinct plausible component of a causal system and was a noteworthy component of prevailing cognitive-behavioral theories of eating disorders. Prior network examinations of eating disorders have used similar item- level measurement (Forbush, Siew, \& Vitevitch, 2016; Levinson et al., 2017). Furthermore, we created a composite score for symptoms that conceptually spanned across multiple items (e.g., overvaluation of shape and overvaluation of weight were combined to represent the symptom overvaluation of shape and weight), and/or for multiple items that held plausibly similar stimulus functions (e.g., purging and excessive exercise representing energy out). Table 1 depicts the symptoms we included in the current study, along with the items used to measure them and, when applicable, the internal consistency.

Our network included 10 eating disorder symptoms, namely: fear of gaining weight, cognitive restraint, dietary restriction, body dissatisfaction, preoccupation with eating and body image, drive for thinness, overvaluation of shape and weight, fear of loss of control over eating, and two symptoms that we termed energy in and energy out. The first of these, energy in, included eating large amounts of food. The second, energy out, included self-induced vomiting and driven exercise. Although these are sometimes referred to as binging and purging behaviors, we chose to describe them as behaviors that increase or expend energy to clarify that these behaviors were not limited to loss of control situations (for intake) or contexts in which they followed binge eating (for expenditure), and to specify that energy out behaviors were indeed limited to those that subjectively or objectively expend energy (as opposed to restricting intake e.g., through fasting, which is also often included in purging behaviors). In addition, given the emphasis placed on the causal relationships between symptoms from a network perspective, our labels aimed to highlight the functions of behaviors within the system. Finally, we aimed to base our investigation on a network structure that could be replicated across samples and timeframes as much as possible. Thus, within our theoretical framework, these behaviors may influence other ED disorder symptoms through cognitive pathways but also through physiological pathways of energy balance. These symptoms were chosen based on the transdiagnostic theory of eating disorders (Fairburn et al., 2003), the separation insofar as possible between behavioral components from inner experiences (thoughts and feelings), as well as the rationale outlined above regarding symptom function and causal relationships.

Table 1

Symptom Measurement, Internal Consistency, and Scaled Contribution to BIC

\begin{tabular}{|c|c|c|c|c|c|}
\hline \multirow[b]{2}{*}{ Symptom } & \multirow[b]{2}{*}{ Measure: Item number } & \multirow[b]{2}{*}{ Cronbach's $\alpha$} & \multicolumn{3}{|c|}{ Scaled contribution to BIC } \\
\hline & & & Full sample & No abuse & Abuse \\
\hline Fear of gaining weight & EDE-Q: 10 & & -.53 & -.31 & .03 \\
\hline Cognitive restraint & EDE-Q: $1,3,4,5$ & .88 & -1.11 & -.61 & -.13 \\
\hline Dietary restriction & EDE-Q: 2 & & .08 & -.70 & .73 \\
\hline Body dissatisfaction & EDE-Q: 25,26 & .86 & -.83 & -.08 & -.48 \\
\hline Loss of control & EDE-Q: 9 & & -.82 & -.42 & .09 \\
\hline Preoccupation with eating and body image & EDE-Q: 7,8 & .84 & -.84 & -1.00 & .01 \\
\hline Energy out & EDE-Q: 16,18 & & .93 & -.13 & 1.10 \\
\hline Energy in & EDE-Q: 13 & & 1.36 & 2.46 & -2.56 \\
\hline Drive for thinness & EDE-Q: 6 & & -.70 & -.39 & -.19 \\
\hline Overvaluation of shape and weight & EDE-Q: 22,23 & .93 & 1.40 & 1.25 & .28 \\
\hline Depressed mood & BDI: 1 & & 1.07 & -.04 & 1.12 \\
\hline
\end{tabular}

Note. EDE-Q $=$ Eating Disorder Examination Questionnaire; BDI $=$ Beck Depression Inventory. 


\section{Childhood Abuse}

Childhood abuse was assessed using the French version of the Childhood Trauma Questionnaire (CTQ; Bernstein \& Fink, 1998), which retrospectively examines childhood abuse through selfreported assessment. Specifically, in this study the sexual abuse, physical abuse, and emotional abuse subscales were used. Scores range from 5 to 25 for each type of abuse. In line with the recommendations of Bernstein and Fink (1998), thresholds or cut-off scores were set for each type of abuse at four levels of maltreatment: none, low, moderate, and severe. Each subtype was then further dichotomized as present (moderate/severe/low) versus absent. Given previous evidence of different effects of abuse and neglect (Guillaume et al., 2016), and recommendations to examine them separately when investigating their relationships with mental health disorders (Norman et al., 2012), the current investigation focused on childhood abuse rather than neglect that has sometimes also been considered to be a form of childhood trauma.

\section{Statistical Analysis}

Our network was primarily grounded in the transdiagnostic theory of eating disorders (Fairburn et al., 2003), and informed by previously identified network structures of eating disorder symptoms (DuBois et al., 2017; Rodgers et al., 2018). Furthermore, consistent with the transdiagnostic theory of eating disorders, as well as the escape theory of the relationship between traumatic experiences and eating disorders (Fairburn et al., 2003; Trottier, Wonderlich, Monson, Crosby, \& Olmsted, 2016), we included negative affect, here measured by depressed mood, as an important component of the network. Of note, consistent with previous work (DuBois et al., 2017; Rodgers et al., 2018), our network did not include body mass index (BMI) for several reasons. First, network theory posits that the constructs represented by different nodes covary across similar time intervals. BMI variations occur on a very different time scale, which makes it unsuitable to include as a node from this perspective. Furthermore, although the effects of malnutrition may play an important role in eating disorder psychopathology, as may experiences of weight-related stigma, these are arguably not a consequence of BMI per se but rather the behaviors and cognitions related to BMI in an individual and social context. Finally, the relationship between BMI and eating disorder symptoms is poorly characterized, but most likely not linear, which makes it less suitable for inclusion in directed network analyses.

Bayesian network analysis. Our Bayesian network analysis consisted of two steps. First, we created a set of rules regarding implausible symptom relationships based on previous clinical models and conceptual understanding of eating disorder psychopathology (e.g., Murphy, Straebler, Cooper, \& Fairburn, 2010). These rules were used to ensure that our model only investigated patterns of symptom relationships that made both conceptual and clinical sense, or that have been hypothesized in previous research. For example, results from a longitudinal network analysis study in eating disorders found that different symptom relationships exist at different temporal intervals (Levinson, Vanzhula, \& Brosof, 2018). As such, a primary consideration in our approach to model specification was the temporal variability of the symptoms included in the model.
As a result, the implausible symptom relationships in our model specify that no symptom could lead to shape and weight overvaluation, resulting in overvaluation as the theoretical top of our Bayesian network model. This approach is further corroborated by the identification in cognitive-behavioral theory of overvaluation of shape and weight as the core mechanism underlying the daily variability in cognitive, affective, and behavioral symptoms comprising eating disorder psychopathology (Murphy et al., 2010). More specifically, in the context of causal systems, the time scale at which overvaluation of weight and shape is hypothesized to vary (over longer periods of time, and lower moment-by-moment variability) is likely to be considerably different than the moment-bymoment variability of the other eating disorder symptoms (greater moment-by-moment variability). As a result, overvaluation of weight and shape plays a unique role in a causal system that is fundamentally different than the other eating disorder symptomsone that is more stable over time and represents the core of the disorder (Fairburn et al., 2003). These rules were integrated into our model such that the implausible symptom relationships would be omitted (hereby referred to as blacklisted) from the learning algorithm described below.

Next, we used a particular type of learning algorithm, called the hill-climbing algorithm, to estimate the structure of the Bayesian networks from our data (Tsamardinos, Brown, \& Aliferis, 2006). The hill-climbing algorithm is an iterative machine-learning process that begins with an arbitrary network structure, then attempts to find a better structure by making incremental changes to the network, such as adding, removing, or reversing edges. If a change produces a better model fit, as measured by a reduction in the Bayesian Information Criterion (BIC), then another incremental change is made to the new network structure, and so on, until no further improvements can be found. Using the hill-climbing algorithm applied to our data, our network models represent the highest possible model fit for the interactive structure of eating disorder psychopathology. This method was applied to the full sample, as well as to the abuse experience and no abuse experience subgroups (defined below), resulting in three separate Bayesian network analyses. In these models, each node represented one of the eight eating disorder symptoms, and each edge represented the predicted relationship between a given pair of symptoms.

Although the hill-climbing algorithm converges on the network structures with the best possible model fit, the degree of confidence we can have when interpreting specific pathways within the networks is unknown. As such, an important consideration in Bayesian network analysis is the quantification of arc strength, representing the degree of confidence we can have in the presence and direction of each individual edge in the network. We measured arc strength through a bootstrapping procedure (Friedman, Goldszmidt, \& Wyner, 1999). First, we generated multiple $(N=2000)$ network structures by applying nonparametric bootstrapping to our data. Next, we calculated the relative frequency of the presence and direction of each edge across the bootstrapped samples. Edges that were frequently present in the bootstrapped networks, and that maintained the same directionality, were considered to have a high arc strength and could reasonably be interpreted with a high degree of confidence. Alternatively, edges that were only present in a small subset of the bootstrapped networks were considered to have low arc strength and should be interpreted with caution. 
Symptom importance. We calculated each symptom's importance based on the relative contribution that each symptom contributed to the overall model fit of the Bayesian network (Scutari, 2016). Symptoms with greater contributions to the overall model fit were conceptualized as important to the eating disorder networks as a whole, whereas symptoms with only minimal contribution to the overall model fit were considered less important to the networks.

Differences in network structure. We investigated differences in symptom importance and overall network structure between individuals with eating disorders with and without a past experience of abuse. First, individuals were grouped based on whether or not they endorsed a past experience of abuse, as measured by the three subscales of the CTQ. Individuals with at least a low to moderate score on any of the three CTQ subscales were placed into the abuse -experience group. Likewise, the remaining individuals with a score of none to minimal on all CTQ subscales were placed into a no abuse experience group. Next, we applied the hill-climbing algorithm, with implausible relationships blacklisted from the model, to both groups, resulting in one Bayesian network structure for those with no past experience of abuse, and one Bayesian network structure for those with a past experience of abuse. We used these two networks to descriptively compare differences in the symptom importance profile between the two groups, as well as to explore differences in the patterns of relationships among eating disorder symptoms between the two groups.

\section{Results}

\section{Descriptive Findings}

The average BMI among the final sample was 20.34 ( $S D=$ 6.30). Forty-six percent of the sample endorsed at least low to moderate experience of abuse during childhood $(n=149)$, whereas $54 \%$ of the sample did not endorse any past abuse experience during childhood $(n=178)$. Among individuals with a past experience of abuse, $89 \%(n=132)$ reported at least low to moderate emotional abuse, $30 \%(n=44)$ reported at least low to moderate physical abuse, and $44 \%(n=66)$ reported at least low to moderate sexual abuse.

\section{Bayesian Network Analysis}

A graphical representation of the full sample Bayesian network is depicted in Figure 1. Applying our implausible symptom relationship rules to the model resulted in two edge omissions (body dissatisfaction leading to overvaluation of shape and weight, and body dissatisfaction leading to fear of gaining weight) when compared with an unrestrained model. ${ }^{1}$ In the final blacklisted model, all symptoms were significantly connected within the network. Overvaluation of shape and weight was the only symptom exhibiting only outgoing edges in the eating disorder network. Energy in (overeating), energy out (self-induced vomiting and driven exercise), dietary restriction, drive for thinness, and loss of control were the five symptoms exhibiting only incoming edges. Furthermore, fear of gaining weight was identified as the symptom with the greatest amount of outgoing edges in the eating disorder network ( $n=5$; fear of gaining weight $\rightarrow$ preoccupation with

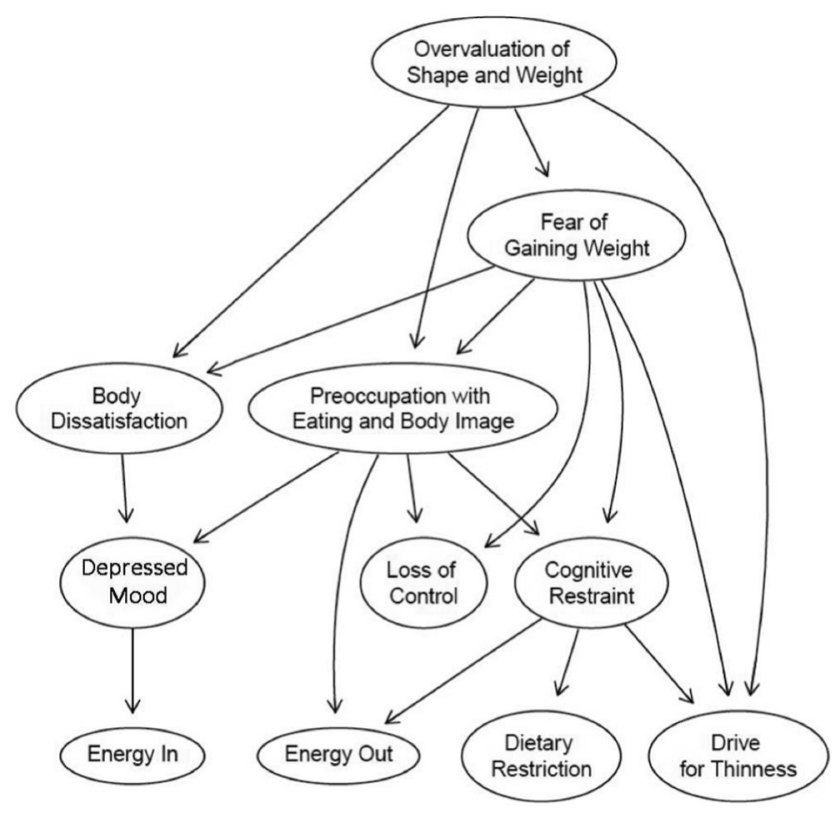

Figure 1. Full-sample Bayesian network structure.

eating and body image, loss of control, drive for thinness, body dissatisfaction, and cognitive restraint), suggesting a broader range of influence when compared with symptom with fewer outgoing edges. Conversely, drive for thinness was identified as the symptom with the greatest amount of incoming edges in the eating disorder network ( $n=3$; overvaluation of shape and weight, cognitive restraint, and fear of gaining weight, $\rightarrow$ drive for thinness).

Noteworthy differences in arc strength within the full sample Bayesian network emerged. The average frequency of the presence of edges correctly identified across bootstrapped samples was $84 \%$, with a maximum frequency of $99 \%$ (fear of gaining weight $\rightarrow$ cognitive restraint) and a minimum frequency of $51 \%$ (preoccupation with eating and body image $\rightarrow$ cognitive restraint). The average frequency of the direction of edges correctly identified across the bootstrapped samples was $63 \%$, with a maximum frequency of $88 \%$ (preoccupation with eating and body image $\rightarrow$ depressed mood) and a minimum frequency of $35 \%$ (preoccupation with eating and body image $\rightarrow$ cognitive restraint).

The profile of symptom importance is presented in Table 1 . Symptoms significantly differed in their relative contribution to the overall Bayesian network model. Overvaluation of shape and weight was identified as the most important symptom in the full sample Bayesian network (BIC contribution $=-466.39$; scaled importance $=1.40$ ), followed by energy in (BIC contribution $=-464.91$; scaled importance $=1.36$ ) and depressed mood $(\mathrm{BIC}$ contribution $=-455.21$; scaled importance $=1.07)$. Con-

\footnotetext{
${ }^{1}$ Results from the same network analysis with no blacklisting resulted in the following structural differences when compared with the final network structure with blacklisting presented in the manuscript: one outward arrow going from body dissatisfaction to overvaluation, an outward arrow going from fear of gaining weight to overvaluation, and an outward arrow from drive for thinness to overvaluation.
} 
versely, cognitive restraint (BIC contribution $=-381.03$; scaled importance $=-1.11$ ) was identified as the least important symptom in the full sample network, followed by preoccupation with eating and body image (BIC contribution $=-390.30$; scaled importance $=-0.84$ ) and body dissatisfaction (BIC contribution $=-390.50 ;$ scaled importance $=-0.83$ ).

\section{Comparison of Abuse and No-Abuse Groups}

Compared with those with no abuse history, individuals with a history of abuse had significantly higher global EDE scores, $t(323)=4.33, p<.01$, and a higher average BMI, $t(314)=2.23$, $p<.05$. Specifically, individuals with no history of abuse displayed an average eating disorder severity in the 85th percentile (global EDE score $=2.99$ ), whereas those with a history of abuse reported an average eating disorder severity in the 90th percentile (global EDE score = 3.69; see Mond, Hay, Rodgers, \& Owen, 2006 for norms among young adult women).

Differences in network structure. Differences in the network structure between individuals with eating disorders with and without a past experience of abuse are visualized in Figure 2, with the structure only within individuals with and without a past experience of abuse represented in Figures 3 and 4, respectively. A total of nine edges were identified as being shared across both groups. However, there were a number of significant differences in the network structure across the two groups. A total of seven edges in the abuse experience eating disorder network were not identified in the no abuse experience eating disorder network, representing symptom relationships that are unique to individuals with eating

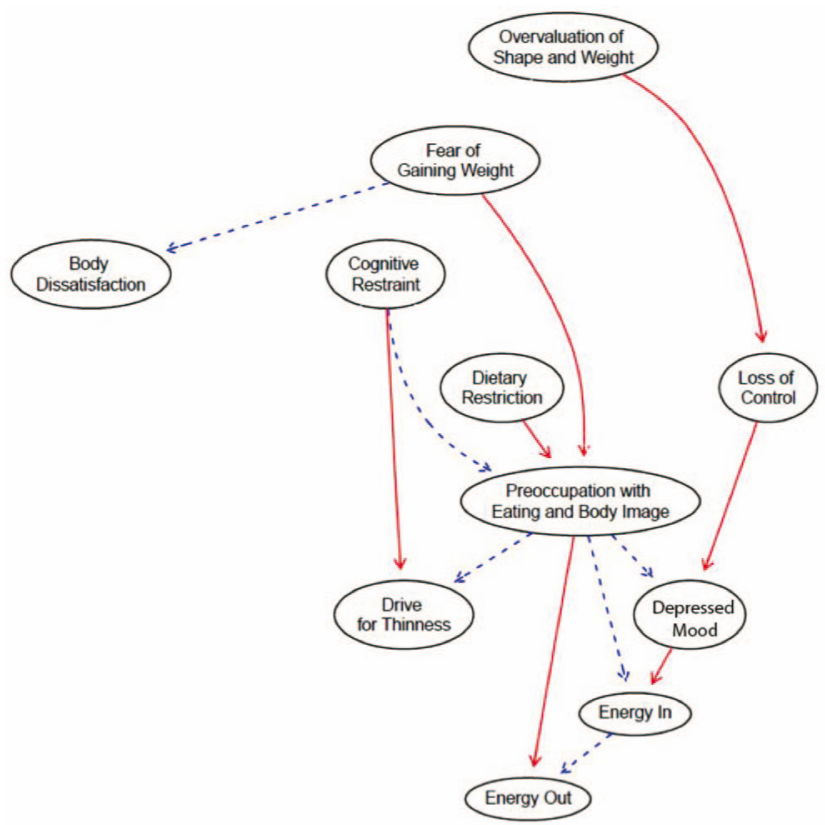

Figure 2. Comparison of symptom relationships. Solid (red in online version only) edges indicate relationships that are unique to individuals with a past experience of abuse. Dotted (blue in online version only) edges indicate relationships that are unique to individuals with no past experience of abuse. Edges shared by both groups are omitted from this network. See the online article for the color version of this figure.

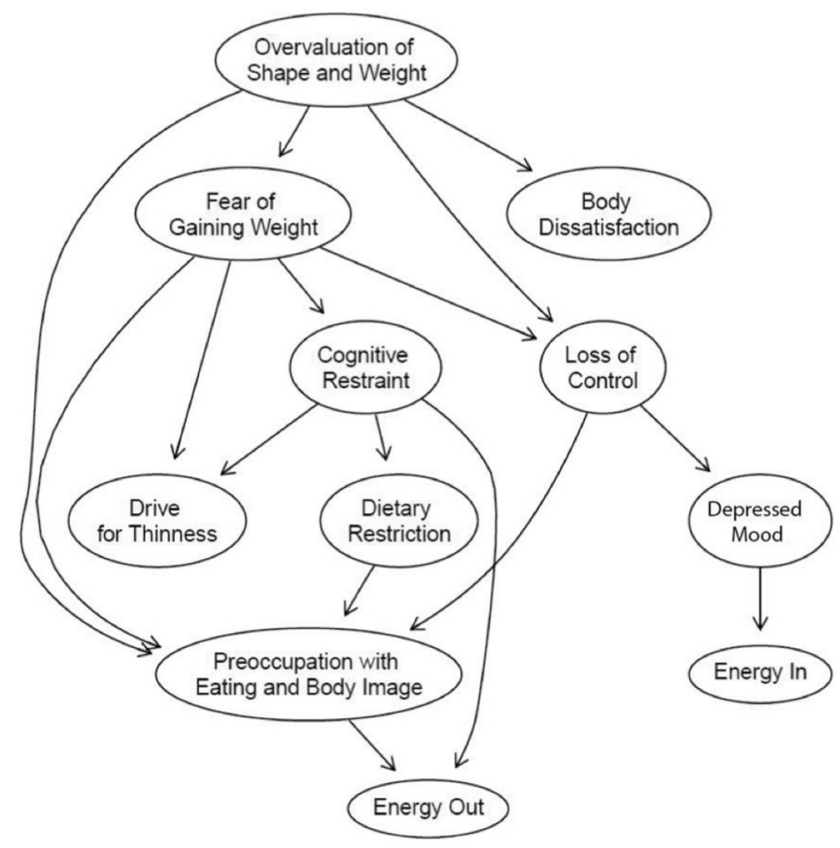

Figure 3. Bayesian network structure for individuals with a past experience of abuse.

disorders with a past experience of abuse. These include fear of gaining weight leading to preoccupation with eating and body image, dietary restriction leading to preoccupation with eating and body image, and cognitive restraint leading to drive for thinness, among others. Furthermore, a specific four-symptom pathway unique to the abuse experience eating disorder network emerged, leading

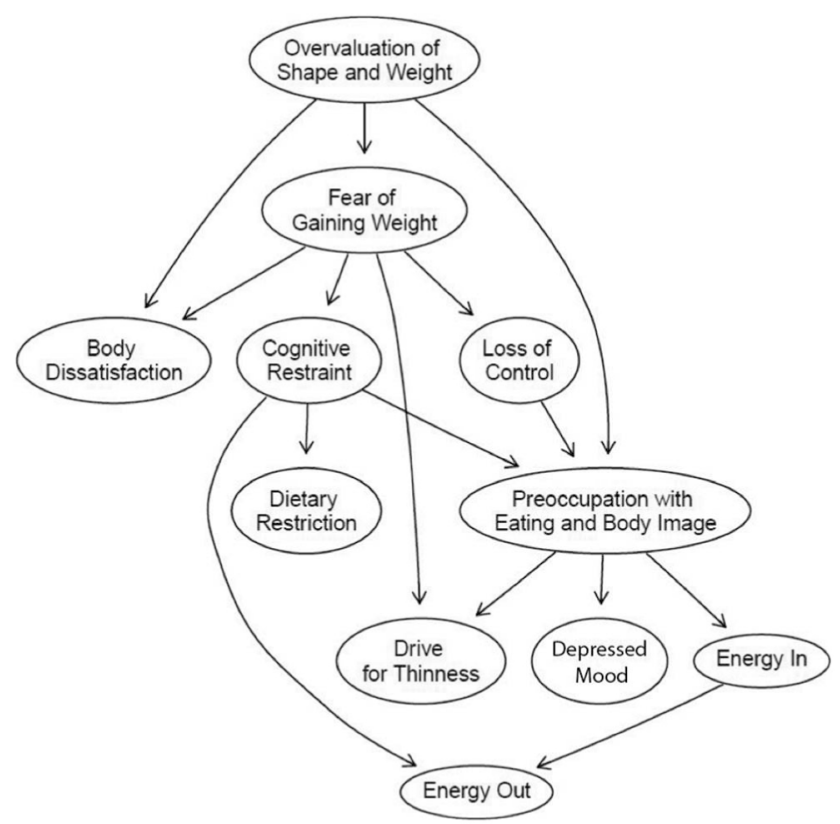

Figure 4. Bayesian network structure for individuals with no past experience of abuse. 
from overvaluation of shape and weight and ending in energy in (overvaluation of weight and shape $\rightarrow$ loss of control $\rightarrow$ depressed mood $\rightarrow$ energy in).

A total of six edges were identified as symptom relationships that were unique only to those who have no past experience of abuse. For example, preoccupation with eating and body image had a much larger predictive role in the no-abuse -experience eating disorder network (three outward arrows) when compared with the abuse-experience eating disorder network (one outward arrow). Other noteworthy pathways unique to the no-abuse -experience eating disorder network include fear of gaining weight leading to body dissatisfaction, preoccupation with eating and body image leading to drive for thinness, and cognitive restraint leading to a preoccupation with eating and body image, among others. Furthermore, a specific four-symptom pathway unique to the no-abuse-experience eating disorder network emerged, leading from cognitive restraint and ending in energy out (cognitive restraint $\rightarrow$ preoccupation with eating and body image $\rightarrow$ energy in $\rightarrow$ energy out).

Differences in symptom importance. Differences in symptom importance between individuals with eating disorders with and without a past experience of abuse are visualized in Table 1. The three symptoms with the highest importance in the no- abuse -experience eating disorder network were the same as those identified in the full sample eating disorder network, albeit in a different order. Energy-in was identified as the most important symptom (BIC contribution $=-297.53$; scaled importance $=2.46$ ), followed by overvaluation (BIC contribution $=-263.70)$ and depressed mood (BIC contribution $=-227.30$; scaled importance $=-0.04$ ). The symptom with the least importance in the no- abuse -experience eating disorder network was preoccupation with eating and body image (BIC contribution $=-200.54$; scaled importance $=-1.00)$, followed by dietary restriction (BIC contribution $=-209.03$; scaled importance $=-0.70$ ) and cognitive restraint (BIC contribution $=-211.45$; scaled importance $=-0.61$ ).

Two of the three most important symptoms in the abuse experience eating disorder network were unique to this group. Specifically, depressed mood was identified as the most important symptom (BIC contribution $=-225.04$; scaled importance $=1.12$ ), followed by energy-out $(\mathrm{BIC}$ contribution $=-224.25$; scaled importance $=1.10$ ) and dietary restriction (BIC contribution $=-210.36$; scaled importance $=0.73$ ). Conversely, the symptom with the least importance in the abuse experience eating disorder network was energy-in (BIC contribution $=-87.93$; scaled importance $=-2.56$ ), followed by body dissatisfaction (BIC contribution $=-165.09$; scaled importance $=-0.48$ ) and cognitive restraint (BIC contribution $=-178.11 ;$ scaled importance $=-0.13$ ).

\section{Discussion}

The aim of this study was to apply a Bayesian network approach to model the interactive network structure of eating disorder psychopathology, and to investigate the differences in symptom importance and overall network structure between individuals with eating disorders with and without an experience of childhood abuse. Overall our findings in the full sample were consistent with those from previous investigations using network approaches regarding important roles of overvaluation of shape and weight, and fear of gaining weight (DuBois et al.,
2017; Rodgers et al., 2018). In addition, our findings pointed to differences in the directed network structures of eating disorder symptoms between individuals with and without a history of childhood abuse. Specifically, among individuals with a history of childhood abuse, loss of control eating and depressed mood emerged as more important driving symptoms of the disorder, with preoccupation with eating and body image emerging as a consequence.

In our entire sample, overvaluation of shape and weight and fear of gaining weight appeared at the top of the network, as the only nodes exhibiting only outgoing edges, consistent with the transdiagnostic theory of eating disorders (Fairburn et al., 2003). Similarly consistent with that theory, fear of gaining weight was associated with preoccupation with eating and body shape, which were in turn related to cognitive restraint and eating disorder behaviors including loss of control eating, dietary restriction, and compensatory behaviors. Furthermore, overvaluation of shape and weight emerged as the most important symptom in the full sample Bayesian network, confirming its key role in driving eating disorder psychopathology, and consistent with findings from previous network studies (DuBois et al., 2017). Interestingly, in the full sample network, fear of gaining weight was identified as the symptom with the greatest amount of outgoing edges, whereas drive for thinness presented incoming edges only, which provides further support for the usefulness of distinguishing between these two constructs and examining their differential contributions to eating disorder psychopathology (Rodgers et al., 2018). In addition, preoccupation with eating and body image displayed the second largest number of outgoing edges, and was associated with depressed mood and cognitive restraint as well as loss of control eating and compensatory behaviors. Recent work examining the respective roles of overvaluation, body dissatisfaction, and preoccupation with weight and shape has supported the distinction of these three dimensions as independent constructs, and suggested that, among females, preoccupation with weight and shape might be most strongly associated with eating disorder behaviors (Mitchison et al., 2017). Our findings align with these previous results, and provide additional support for role of preoccupation in subsequent eating disorder behaviors.

Our findings identified two distinct eating disorder symptoms networks among individuals with and without a history of childhood abuse, differing both in the patterns of relationships among symptoms, as well as the relative importance of specific symptoms, and thus supporting the idea that the processes and mechanisms underlying the development and maintenance of eating disorder pathology among individuals with a abuse history might be specific to this group. Among individuals with a history of childhood abuse, and consistent with escape theory (Trottier et al., 2016), depressed mood emerged as an important driver of eating disorder psychopathology. Indeed, a unique and specific foursymptom pathway emerged, leading from overvaluation of shape and weight, to loss of control, depressed mood, and overeating. Furthermore, and in contrast to the network among individuals without a history of childhood abuse, depressed mood was identified as the most important symptom in the network. In addition, preoccupation with eating and body image displayed only one outgoing edge in the network specific to this group, and was of low importance to the network, as was overvaluation of weight and shape. 
Among the participants with no history of childhood abuse, the directed network identified presented a number of different characteristics. First, it was characterized by six unique edges, representing pathways present only among this group. Most notably, as mentioned above, preoccupation with eating and body image played much larger predictive role in the no abuse experience eating disorder network. In addition. the symptoms with the highest importance in this group were overeating (energy in), and overvaluation of weight and shape. Thus, the relationships and structure of the eating disorder symptom network among the group with no history of childhood abuse was more clearly consistent with transdiagnostic theories (Fairburn et al., 2003), organized around a heightened investment in weight and shape, and resulting efforts to control or alter weight and shape through dieting and exercise behaviors.

These findings have a number of clinical implications. In other types of psychopathology such as depression, anxiety, and substance use disorder, the phenotypic expression of psychopathology may be strongly influenced by abuse and trauma, and the concept of a maltreated eco-phenotype that might represent distinct subtypes of a given disorder has been introduced (Teicher \& Samson, 2013). Recent finding have suggested that this might be a useful construct to consider in the context of eating disorders (Monteleone, Patriciello, et al., 2018). The current findings are consistent with the existence of such different phenotypic expressions, and suggest the usefulness of using this framework to guide our understanding of psychopathology among individuals with a history of trauma.

In addition, as described above, the complex psychopathological features, poorer outcomes, and high drop-out rates that characterize individual with eating disorders with a history of childhood abuse pose particular clinical challenges (Castellini et al., 2018). The distinct eating disorder symptom network characteristic of eating pathology in the presence of a history of children abuse suggested by our results may help to inform treatment strategies. Given the central role played by depressed mood in the symptom network among individuals with childhood abuse, and, in contrast the lesser importance of body image related dimensions including overvaluation of weight and shape, it may be that treatments approaches centered upon emotional regulation and might be most helpful among this group (Sloan et al., 2017). Third-wave behavior therapies may be particularly well suited for example, as they target response-focused emotion regulation strategies (i.e., strategies that modulate the expression or experience of emotion regulation after its initiation; Hayes, Villatte, Levin, \& Hildebrandt, 2011). Currently, third-wave therapies do not meet established criteria for an empirically supported treatment for eating disorder (Linardon, Fairburn, Fitzsimmons-Craft, Wilfley, \& Brennan, 2017). Nevertheless, we speculate that they might be useful, specifically in the subgroup of patients with high levels of emotional disturbance such as those with childhood abuse.

Our study has several limitations. Related to the measurement of childhood trauma, our study is limited by the use of retrospective self-report measures of childhood abuse. It cannot be ruled out that some participants may have difficulty recalling events of their early years or may have chosen not to disclose their maltreatment. In addition, several important factors can modify the effects of abuse, including the frequency and co-occurrence of multiple types of abuse and other trauma, or the developmental period during which it when it occurs (Tyrka, Burgers, Philip, Price, \& Carpenter, 2013), and our measure was not able to distinguish childhood trauma along these characteristics. It should nevertheless be noted that despite its limitations, the CTQ is a widely used and statistically robust measure. Likewise, we considered a collapsed measure of childhood abuse that, while distinguishing it from childhood trauma, did not allow us to examine the additive impact of multiple trauma, nor its dose-dependent relationship with eating pathology. In addition, some of our network nodes utilized single items while others used composites. An additional limitation may be related to our sample that was diagnostically heterogeneous, although this does allow for the examination of transdiagnostic models.

Furthermore, it is important to consider a number of limitations related to our Bayesian analytic approach. One, it is not known whether the differences that were found in symptom importance were statistically significant, and the differences described are therefore descriptive. Alternative network analytic techniques, such as the network comparison test of structural invariance and centrality difference test for weighted undirected concentration networks, may be better suited for direct statistical comparison of two (or more) network structures. Two, although our network analytic approach produced directed edges, it is not known the extent to which directionality in each edge corresponds to true causal relations. For example, Bayesian models assume that all key variables are included in the model. It may be that certain variables, if added to our network model, may have produced different results. Three, it is reasonable to conclude that certain eating disorder symptoms do, in fact, influence shape and weight overvaluation, despite the fact that they were blacklisted in our model. However, in relation to causal systems, the longer time scale over which overvaluation of weight and shape is hypothesized to vary is likely to be considerably different to the moment-by-moment variability of the other eating disorder symptoms. As a result, overvaluation of weight and shape plays a unique role in a causal system that is fundamentally different than the other eating disorder symptoms one that is more stable and represents the core of the disorder (Fairburn et al., 2003). Despite this difference, future research is indeed needed to better understand the impact of differential temporal variability on psychopathology networks. A last limitation of this approach is its failure to represent bidirectional relationships and feedback loops within networks. Future work should consider using for example intensive longitudinal data and other analytic approaches that are better suited to capture these.

Despite these limitations, our study presents novel and important findings supporting the existence of a distinct eating disorder symptom network, characteristic of individuals with a history of childhood abuse. Further research from this perspective will be helpful in refining our understanding of the specificities of eating disorder psychopathology in individuals with a history of childhood trauma. Useful avenues of investigation might include examining other network characteristics, such as the connectivity of symptom network among individuals with a history of childhood trauma, which provides an index of the strengths of the relationships among different symptoms. It has been suggested that childhood trauma might increase the risk of eating disorders, through general heightened susceptibility to psychopathology, in addition to the existence of heightened risk specific to the effects of trauma (Holzer, Uppala, Wonderlich, Crosby, \& Simonich, 2008). Fur- 
thermore, examining the functional pathways shared between symptoms of trauma-related disorders, such as PTSD, and symptoms of eating disorders may elucidate the mechanisms underlying comorbidity between these two areas of psychopathology. Further research grounded in the network approach may continue to help to clarify these questions.

\section{References}

Beck, A. T., Steer, R. A., \& Carbin, M. G. (1988). Psychometric properties of the Beck Depression Inventory: Twenty-five years of evaluation. Clinical Psychology Review, 8, 77-100. http://dx.doi.org/10.1016/02727358(88)90050-5

Bernstein, D. P., \& Fink, L. (1998). Childhood trauma questionnaire: A retrospective self-report: Manual. San Antonio, TX: Psychological Corporation.

Borsboom, D., \& Cramer, A. O. (2013). Network analysis: An integrative approach to the structure of psychopathology. Annual Review of Clinical Psychology, 9, 91-121. http://dx.doi.org/10.1146/annurev-clinpsy-050212185608

Brewerton, T. D. (2007). Eating disorders, trauma, and comorbidity: Focus on PTSD. Eating Disorders, 15, 285-304. http://dx.doi.org/10.1080/ 10640260701454311

Caslini, M., Bartoli, F., Crocamo, C., Dakanalis, A., Clerici, M., \& Carrà, G. (2016). Disentangling the association between child abuse and eating disorders: A systematic review and meta-analysis. Psychosomatic Medicine, 78, 79-90. http://dx.doi.org/10.1097/PSY.0000000000000233

Castellini, G., Lelli, L., Cassioli, E., Ciampi, E., Zamponi, F., Campone, B., . . Ricca, V. (2018). Different outcomes, psychopathological features, and comorbidities in patients with eating disorders reporting childhood abuse: A 3-year follow-up study. European Eating Disorders Review, 26, 217-229. http://dx.doi.org/10.1002/erv.2586

DuBois, R. H., Rodgers, R. F., Franko, D. L., Eddy, K. T., \& Thomas, J. J. (2017). A network analysis investigation of the cognitive-behavioral theory of eating disorders. Behaviour Research and Therapy, 97, 213221. http://dx.doi.org/10.1016/j.brat.2017.08.004

Fairburn, C. G., Cooper, Z., \& Shafran, R. (2003). Cognitive behaviour therapy for eating disorders: A "transdiagnostic" theory and treatment. Behaviour Research and Therapy, 41, 509-528. http://dx.doi.org/10.1016/ S0005-7967(02)00088-8

Fairburn, S. G., \& Beglin, S. J. (2008). Cognitive behavior therapy and eating disorders. New York, NY: Guilford Press.

Forbush, K. T., Siew, C. S., \& Vitevitch, M. S. (2016). Application of network analysis to identify interactive systems of eating disorder psychopathology. Psychological Medicine, 46, 2667-2677. http://dx.doi.org/10.1017/ S003329171600012X

Fried, E. I., van Borkulo, C. D., Cramer, A. O. J., Boschloo, L., Schoevers, R. A., \& Borsboom, D. (2017). Mental disorders as networks of problems: A review of recent insights. Social Psychiatry and Psychiatric Epidemiology, 52, 1-10. http://dx.doi.org/10.1007/s00127-016-1319-z

Friedman, N., Goldszmidt, M., \& Wyner, A. (1999). Data analysis with Bayesian networks: A bootstrap approach. In UAI '99: Proceedings of the 15th Annual Conference on Uncertainty in Artificial Intelligence (pp. 196-205). Burlington, MA: Morgan Kaufmann.

Guillaume, S., Jaussent, I., Maimoun, L., Ryst, A., Seneque, M., Villain, L., . . . Courtet, P. (2016). Associations between adverse childhood experiences and clinical characteristics of eating disorders. Scientific Reports, 6, 35761. http://dx.doi.org/10.1038/srep35761

Harrington, E. F., Crowther, J. H., Payne Henrickson, H. C., \& Mickelson, K. D. (2006). The relationships among trauma, stress, ethnicity, and binge eating. Cultural Diversity and Ethnic Minority Psychology, 12, 212-229. http://dx.doi.org/10.1037/1099-9809.12.2.212

Hayes, S. C., Villatte, M., Levin, M., \& Hildebrandt, M. (2011). Open, aware, and active: Contextual approaches as an emerging trend in the behavioral and cognitive therapies. Annual Review of Clinical Psychology, 7, 141168. http://dx.doi.org/10.1146/annurev-clinpsy-032210-104449

Haynos, A. F., \& Fruzzetti, A. E. (2011). Anorexia nervosa as a disorder of emotion dysregulation: Evidence and treatment implications. Clinical Psychology: Science and Practice, 18, 183-202. http://dx.doi.org/10.1111/j .1468-2850.2011.01250.x

Heckerman, D., Geiger, D., \& Chickering, D. M. (1995). Learning Bayesian networks: The combination of knowledge and statistical data. Machine Learning, 20, 197-243. http://dx.doi.org/10.1007/BF00994016

Holzer, S. R., Uppala, S., Wonderlich, S. A., Crosby, R. D., \& Simonich, H. (2008). Mediational significance of PTSD in the relationship of sexual trauma and eating disorders. Child Abuse \& Neglect, 32, 561566. http://dx.doi.org/10.1016/j.chiabu.2007.07.011

Jones, P. J., Mair, P., Riemann, B. C., Mugno, B. L., \& McNally, R. J. (2018). A network perspective on comorbid depression in adolescents with obsessive-compulsive disorder. Journal of Anxiety Disorders, 53, 1-8. http://dx.doi.org/10.1016/j.janxdis.2017.09.008

Lecrubier, Y., Sheehan, D. V., Weiller, E., Amorim, P., Bonora, I., Sheehan, K. H., . . Dunbar, G. C. (1997). The Mini International Neuropsychiatric Interview (MINI). A short diagnostic structured interview: Reliability and validity according to the CIDI. European Psychiatry, 12, 224-231. http://dx.doi.org/10.1016/S0924-9338(97)83296-8

Levinson, C. A., Vanzhula, I., \& Brosof, L. C. (2018). Longitudinal and personalized networks of eating disorder cognitions and behaviors: Targets for precision intervention a proof of concept study. International Journal of Eating Disorders, 51, 1233-1243. http://dx.doi.org/10.1002/ eat.22952

Levinson, C. A., Zerwas, S., Calebs, B., Forbush, K., Kordy, H., Watson, H., . . Bulik, C. M. (2017). The core symptoms of bulimia nervosa, anxiety, and depression: A network analysis. Journal of Abnormal Psychology, 126, 340-354. http://dx.doi.org/10.1037/abn0000254

Linardon, J., Fairburn, C. G., Fitzsimmons-Craft, E. E., Wilfley, D. E., \& Brennan, L. (2017). The empirical status of the third-wave behaviour therapies for the treatment of eating disorders: A systematic review. Clinical Psychology Review, 58, 125-140. http://dx.doi.org/10.1016/j.cpr.2017.10 .005

McNally, R. J. (2016). Can network analysis transform psychopathology? Behaviour Research and Therapy, 86, 95-104. http://dx.doi.org/10.1016/j .brat.2016.06.006

McNally, R. J., Heeren, A., \& Robinaugh, D. J. (2017). A Bayesian network analysis of posttraumatic stress disorder symptoms in adults reporting childhood sexual abuse. European Journal of Psychotraumatology, 8(sup. 3), 1341276.

McNally, R. J., Mair, P., Mugno, B. L., \& Riemann, B. C. (2017). Co-morbid obsessive-compulsive disorder and depression: A Bayesian network approach. Psychological Medicine, 47, 1204-1214. http://dx .doi.org/10.1017/S0033291716003287

Michopoulos, V., Powers, A., Moore, C., Villarreal, S., Ressler, K. J., \& Bradley, B. (2015). The mediating role of emotion dysregulation and depression on the relationship between childhood trauma exposure and emotional eating. Appetite, 91, 129-136. http://dx.doi.org/10.1016/j.appet .2015 .03 .036

Mitchison, D., Hay, P., Griffiths, S., Murray, S. B., Bentley, C., GratwickSarll, K., . . Mond, J. (2017). Disentangling body image: The relative associations of overvaluation, dissatisfaction, and preoccupation with psychological distress and eating disorder behaviors in male and female adolescents. International Journal of Eating Disorders, 50, 118-126. http://dx.doi.org/10.1002/eat.22592

Molendijk, M. L., Hoek, H. W., Brewerton, T. D., \& Elzinga, B. M. (2017). Childhood maltreatment and eating disorder pathology: A systematic review and dose-response meta-analysis. Psychological Medicine, 47, 1402-1417. http://dx.doi.org/10.1017/S0033291716003561

Mond, J. M., Hay, P. J., Rodgers, B., \& Owen, C. (2006). Eating Disorder Examination Questionnaire (EDE-Q): Norms for young adult women. 
Behaviour Research and Therapy, 44, 53-62. http://dx.doi.org/10.1016/ j.brat.2004.12.003

Monteleone, A. M., Monteleone, P., Esposito, F., Prinster, A., Ruzzi, V., Canna, A., . . Maj, M. (2017). The effects of childhood maltreatment on brain structure in adults with eating disorders. The World Journal of Biological Psychiatry, 20, 301-309.

Monteleone, A. M., Monteleone, P., Volpe, U., De Riso, F., Fico, G., Giugliano, R., . . Maj, M. (2018). Impaired cortisol awakening response in eating disorder women with childhood trauma exposure: Evidence for a dose-dependent effect of the traumatic load. Psychological Medicine, 48, 952-960. http://dx.doi.org/10.1017/S0033291717002409

Monteleone, A. M., Patriciello, G., Ruzzi, V., Cimino, M., Giorno, C. D., Steardo, L., Jr., . . . Maj, M. (2018). Deranged emotional and cortisol responses to a psychosocial stressor in anorexia nervosa women with childhood trauma exposure: Evidence for a "maltreated ecophenotype"? Journal of Psychiatric Research, 104, 39-45. http://dx.doi.org/10.1016/ j.jpsychires.2018.06.013

Moulton, S. J., Newman, E., Power, K., Swanson, V., \& Day, K. (2015). Childhood trauma and eating psychopathology: A mediating role for dissociation and emotion dysregulation? Child Abuse \& Neglect, 39, 167-174. http://dx.doi.org/10.1016/j.chiabu.2014.07.003

Murphy, R., Straebler, S., Cooper, Z., \& Fairburn, C. G. (2010). Cognitive behavioral therapy for eating disorders. Psychiatric Clinics of North America, 33, 611-627. http://dx.doi.org/10.1016/j.psc.2010.04.004

Norman, R. E., Byambaa, M., De, R., Butchart, A., Scott, J., \& Vos, T. (2012). The long-term health consequences of child physical abuse, emotional abuse, and neglect: A systematic review and meta-analysis. PLoS Medicine, 9, e1001349. http://dx.doi.org/10.1371/journal.pmed.1001349

Pearl, J. (2011). The mathematics of causal relations. In P. E. Shrout, K. M. Keyes, \& K. Ornstein (Eds.), Causality and psychopathology: Finding the determinants of disorders and their cures (pp. 47-65). Oxford, UK: Oxford University Press.

Rodgers, R. F., DuBois, R., Frumkin, M. R., \& Robinaugh, D. J. (2018). A network approach to eating disorder symptomatology: Do desire for thinness and fear of gaining weight play unique roles in the network? Body Image, 27, 1-9. http://dx.doi.org/10.1016/j.bodyim.2018.07.004

Scutari, M. (2016). An empirical-Bayes score for discrete Bayesian networks. Journal of Machine Learning Research, 52, 438-448.
Sloan, E., Hall, K., Moulding, R., Bryce, S., Mildred, H., \& Staiger, P. K. (2017). Emotion regulation as a transdiagnostic treatment construct across anxiety, depression, substance, eating and borderline personality disorders: A systematic review. Clinical Psychology Review, 57, 141163. http://dx.doi.org/10.1016/j.cpr.2017.09.002

Steiger, H., Bruce, K., Gauvin, L., Groleau, P., Joober, R., Israel, M., . . Kin, F. N. Y. (2011). Contributions of the glucocorticoid receptor polymorphism (Bcl1) and childhood abuse to risk of bulimia nervosa. Psychiatry Research, 187, 193-197. http://dx.doi.org/10.1016/j.psychres .2010.10.021

Steiger, H., Labonté, B., Groleau, P., Turecki, G., \& Israel, M. (2013). Methylation of the glucocorticoid receptor gene promoter in bulimic women: Associations with borderline personality disorder, suicidality, and exposure to childhood abuse. International Journal of Eating Disorders, 46, 246-255. http://dx.doi.org/10.1002/eat.22113

Teicher, M. H., \& Samson, J. A. (2013). Childhood maltreatment and psychopathology: A case for ecophenotypic variants as clinically and neurobiologically distinct subtypes. The American Journal of Psychiatry, 170, 1114-1133. http://dx.doi.org/10.1176/appi.ajp.2013.12070957

Trottier, K., \& MacDonald, D. E. (2017). Update on psychological trauma, other severe adverse experiences and eating disorders: State of the research and future research directions. Current Psychiatry Reports, 19, 45. http://dx.doi.org/10.1007/s11920-017-0806-6

Trottier, K., Wonderlich, S. A., Monson, C. M., Crosby, R. D., \& Olmsted, M. P. (2016). Investigating posttraumatic stress disorder as a psychological maintaining factor of eating disorders. International Journal of Eating Disorders, 49, 455-457. http://dx.doi.org/10.1002/eat.22516

Tsamardinos, I., Brown, L. E., \& Aliferis, C. F. (2006). The max-min hill-climbing Bayesian network structure learning algorithm. Machine Learning, 65, 31-78. http://dx.doi.org/10.1007/s10994-006-6889-7

Tyrka, A. R., Burgers, D. E., Philip, N. S., Price, L. H., \& Carpenter, L. L. (2013). The neurobiological correlates of childhood adversity and implications for treatment. Acta Psychiatrica Scandinavica, 128, 434-447. http://dx.doi.org/10.1111/acps.12143 